\title{
Exclusion Statistics in a two-dimensional trapped Bose gas
}

\author{
S. Viefers \\ Department of Applied Physics, Chalmers University of Technology and Göteborg University \\ SE-412 96 Göteborg, Sweden
}

T. H. Hansson

Physics Department, Stockholm University

Stockholm Center for Physics, Astronomy and Biotechnology

Roslagstullsbacken 21, S-106 91, Stockholm, Sweden

J. M. Leinaas

Department of Physics, University of Oslo

P.O. Box 1048 Blindern, N-0316 Oslo, Norway

(November 9, 2018)

\begin{abstract}
We briefly explain the notion of exclusion statistics and in particular discuss the concept of an ideal exclusion statistics gas. We then review a recent work where it is demonstrated that a two-dimensional Bose gas with repulsive delta function interactions obeys ideal exclusion statistics, with a fractional parameter related to the interaction strength.
\end{abstract}

PACS numbers: 05.30.Pr, 05.30.Jp, 03.75.Fi 


\section{INTRODUCTION}

Low-dimensional electron systems have been extensively studied during the last decades, both due to their practical importance and because of their exotic quantum mechanical properties. Prominent examples are the 2D heterojunctions that at low temperature, and in high magnetic fields, exhibit the quantum Hall effect [1], and carbon nanotubes, which are quasi $1 \mathrm{~d}$ and (for certain geometries) are expected to behave as Luttinger liquids [2].

At the same time there has been a tremendous advance in the techniques for trapping and cooling atoms, which has opened new vistas for the study of quantum gases, and in particular it is now possible to create quasi-two dimensional atomic Bose gases.

A very interesting property of certain low-dimensional systems is that the elementary excitations obey exotic quantum statistics. The most celebrated example is the quantum Hall effect, where the Laughlin quasi particles are anyons [3], i.e. particles whose quantum statistics "interpolates" between that of bosons and fermions in the sense that their manybody wave function picks up a general phase $\exp (i \theta)$ (rather than just 1 or -1 ) under particle exchange.

Some years ago, Haldane proposed another type of generalized quantum statistics, socalled (fractional) exclusion statistics (FES) [4. Rather than modifying the many-body exchange phases, he generalized the Pauli principle, i.e. introduced new rules for occupying single-particle quantum states. The basic idea of FES is that adding a number of particles, $\Delta N$, to a system, blocks $\Delta d$ of the states available for the next particle according to the linear relation $\Delta d=-g \Delta N$. This corresponds to a repulsion between the particles in phase space, and only very special types of interactions give rise to this type of exclusion of single particle states. One of Haldane's original examples was a one-dimensional spin chain; moreover, he showed that the Laughlin quasi particles (or generally anyons in a strong magnetic field, i.e. confined to the lowest Landau level) also obey exclusion statistics with an exclusion parameter $g=\theta / \pi$. While anyons for topological reasons can exist only in one or two dimensions, there is no such restriction on exclusion statistics particles. Nevertheless, until some time ago, all established examples of FES were either strictly one-dimensional [5.6], or, as anyons in the lowest Landau level, effectively one-dimensional [7 9].

In a recent work [10] we established that within a certain (experimentally feasible) parameter range a (quasi-) two-dimensional Bose gas with repulsive delta function interaction is in fact an ideal FES gas where the exclusion parameter is determined by the interaction strength.

The model Hamiltonian, given by

$$
H=\sum_{i=1}^{N}\left(\frac{p_{i}^{2}}{2 m}+V\left(\vec{r}_{i}\right)\right)+\frac{\pi \hbar^{2}}{m} g \sum_{i<j}^{N} \delta^{2}\left(\vec{r}_{i}-\vec{r}_{j}\right),
$$

has previously been used to describe atoms in Bose condensation experiments using highly asymmetric traps [11,12]. The particular parametrization of the delta function potential is chosen as to reproduce the s-wave scattering phase-shift in three dimensions, and the dimensionless coupling $g$ is given by

$$
g=2 \sqrt{\frac{2}{\pi}} \frac{a}{l_{z}}
$$


where $a$ is the (three dimensional) scattering length and $l_{z}$ the out of plane extension of the asymmetric trap, which is harmonic in the transverse direction with a frequency $\omega=\hbar / m l_{z}^{2}$ [11,13]. We shall assume that the temperature is sufficiently high above the transition temperature that the only relevant mean field is the density, $n$, and that the fluctuations are small enough to be ignored. We shall return to these issues in the discussion (section V).

Our study was motivated by the previous observation [14,13] that, in a Thomas-Fermi approximation, a two dimensional Fermi or Bose gas with short range repulsive interactions has the same energy and number density as an ideal FES gas (treated in the same approximation). To reach our conclusion, we analyzed the statistical mechanics of the delta function gas, both by using a mean field approximation for the energy levels, and by directly relating the thermodynamics to the quantum mechanical scattering phase shifts.

In order to make this presentation reasonably self contained, section 【 gives a brief introduction to exclusion statistics and in particular to ideal FES gases. Section III contains the mean field argument for the equivalence of (1) to an ideal FES gas, and in section [V we show that this result is consistent with a full quantum mechanical treatment of the model within a certain parameter regime. Finally, we discuss our results and point out some open questions.

\section{EXCLUSION STATISTICS}

As already mentioned, exclusion statistics with statistics parameter $g$ is defined by the relation [4]

$$
\Delta d(N)=-g \Delta N
$$

stating that $d(N)$, the dimension of the single-particle Hilbert space when $N-1$ states are occupied, decreases by $g$ whenever a particle is added to the system. Obviously, $g=1$ corresponds to fermions (Pauli blocking), while $g=0$ describes Bose statistics. In general, $g$ can be any rational or integer number. The corresponding statistical weight, i.e. the number of ways in which a given number of particles can be distributed over a given number of states, is 15,16

$$
W=\frac{[D-(g-1)(N-1)] !}{N ![D-g N+g-1] !}
$$

where $D=d(1)$. Eq. (44) is seen to interpolate between the familiar expressions for bosons $(g=0)$ and fermions $(g=1)$ in accordance with Eq.(3).

An ideal FES gas is defined [15] by demanding that this generalized exclusion principle applies locally in energy space i.e. to a set of degenerate or almost degenerate energy levels. In the case of the quantum Hall effect, $g$ is, for topological reasons always rational while in our case, being related to the interaction strength, it can take any real value. For non rational $g, W$ in (雨) is not integer, and has no combinatorical interpretation, but is rather used to define the concept of FES.

In direct analogy with the case of bosons and fermions, the statistical distribution function is derived from (田) by maximizing the non-equilibrium entropy $S \equiv-k_{B} \sum_{k} \ln W_{k}$, 
where the sum is over all energy levels $\epsilon_{k}$, subject to the constraints of keeping the total energy and number of particles fixed [15, 16]. The entropy is found to be

$$
S=\sum_{k} D_{k}\left[\left(1-(g-1) n_{k}\right) \ln \left(1-(g-1) n_{k}\right)-n_{k} \ln n_{k}-\left(1-g n_{k}\right) \ln \left(1-g n_{k}\right)\right],
$$

where $n_{k} \equiv N_{k} / D_{k}$, and the resulting statistical distribution function (or occupancy factor) can be expressed as [15 17] $n_{k}=\left(w_{k}+g\right)^{-1}$ where

$$
w_{k}^{g}\left(1+w_{k}\right)^{1-g}=\exp \left(\beta\left(\epsilon_{k}-\mu\right)\right)
$$

with $\beta=1 / k_{B} T$. The special cases $g=0,1$ correspond to the familiar distribution functions of ideal bosons and fermions, respectively. At zero temperature $n_{k}$ is a step function,

$$
n_{K}^{0}=\frac{1}{g} \theta\left(\epsilon_{F}-\epsilon_{k}\right)
$$

where $\epsilon_{F}$ is the Fermi energy. This means that in the ground state, there are on average $1 / g$ particles in each original quantum state. In section [II], we shall derive the expression (5) for the entropy starting from the 2D delta function gas (11).

The thermodynamics of exclusion statistics has been studied in a number of papers 16 19. A result that is particularly interesting for our purposes, is the virial expansion (i.e. the expansion of the pressure in powers of the density) of an ideal FES gas. As shown by Isakov et al. [17], the equation of state can be evaluated exactly in the special case $D=\sigma$ where $D$ is the number of dimensions and $\sigma$ the power of the dispersion, $\varepsilon(p) \propto p^{\sigma}$. Obviously the case we are interested in, a non-relativistic two dimensional gas with $\varepsilon(p)=p^{2} / 2 m$, falls into this class. Thus, for $D=\sigma=2$, the virial expansion of an ideal FES gas takes the form

$$
\begin{aligned}
\frac{P}{k_{B} T} & =n+\frac{1}{4}(2 g-1) \lambda_{T}^{2} n^{2}+\frac{1}{\lambda_{T}^{2}} \sum_{p=3}^{\infty} \frac{B_{p-1}}{p !}\left(\lambda_{T}^{2} n\right)^{p} \\
& =\frac{P_{\text {Bose }}}{k_{B} T}+\frac{1}{2} g \lambda_{T}^{2} n^{2}
\end{aligned}
$$

where $\lambda_{T}=\sqrt{2 \pi \hbar^{2} \beta / m}$ is the thermal wavelength. Here it is also worth mentioning that for ideal quantum gases in 2D (bosons, fermions or "exclusons"), the pressure equals the energy density $\mathcal{E}$. Note that all statistics dependence in (8) lies in the second virial coefficient, that interpolates linearly between the familiar results for ideal bosons and fermions in 2D [20]. In section $[\mathrm{IV}$ we shall derive such a linear relation for the $2 \mathrm{D}$ delta function gas.

\section{MEAN FIELD THEORY}

Before going into a statistical mechanical analysis of our model, we give a simple thermodynamic mean field argument for why we expect exclusion statistics. For simplicity we consider the case with a constant external potential $V$, so that the density, $n$, is also constant. The crucial observation is then that in a mean field approximation, and for a fixed number of particles, the interaction energy term in (1) just amounts to a constant shift of the energy density, 


$$
\mathcal{E}=\mathcal{E}_{\text {FreeBose }}+\frac{\pi \hbar^{2}}{m} g n^{2} .
$$

Expressing this in terms of the thermal wavelength, we see that this shift is identical to that in the FES virial expansion Eq.(8) if we identify the interaction strength $g$ with the exclusion statistics parameter. In other words, our interacting Bose system (1) has the same (mean field) energy density as an ideal FES gas with statistics $g$.

Let us now consider the statistical mechanics of (11), and assume that the potential $V$ is slowly varying compared with the thermal wavelength, $\lambda_{T}$. We then divide the system into cells of area $b^{2}$, where $\lambda_{T} \ll b \ll|\vec{\nabla} V / V|$, and study the statistical mechanics in each cell. In a mean field approximation, the one-body Hamiltonian in the cell $\ell$ becomes

$$
H_{\ell}=\frac{p^{2}}{2 m}+V\left(\vec{r}_{\ell}\right)+\frac{2 \pi \hbar^{2}}{m} g n\left(\vec{r}_{\ell}\right),
$$

with the corresponding energy $\epsilon^{\ell}$, where we approximate the potential in the cell with the constant $V\left(\vec{r}_{\ell}\right)$ with $\vec{r}_{\ell}$ the position of the center of the cell. Also $n\left(\vec{r}_{\ell}\right)=N_{\ell} / b^{2}$ is the mean number density in the cell $\ell$, with $N_{\ell} \gg 1$ the corresponding average number of particles. 1 We want to demonstrate that the number of available single-particle states in a box $\ell$ decreases with the number of particles as in Haldane's definition of FES, Eq.(3). To this end, we write the quantized momenta in the box as $\vec{p}=(2 \pi \hbar / b) \vec{l}$ with $l_{x}$ and $l_{y}$ integers. The total number of states $d_{\ell}$ below some energy $\epsilon^{\ell}$ corresponding to a momentum $p$, is $d_{\ell}=\pi l^{2}$. Using (11) to eliminate $p$, one finds

$$
\begin{aligned}
d_{\ell} & =\frac{m b^{2}}{2 \pi \hbar^{2}}\left[\epsilon^{\ell}-V\left(r_{\ell}\right)\right]-g N_{\ell} \\
& =d_{\ell, \text { Bose }}-g N_{\ell} .
\end{aligned}
$$

Indeed this result indicates exclusion statistics. Note that an important ingredient of the derivation of $d_{\ell}$ is that the dimension matches the power of the dispersion, in this case $D=\sigma=2$.

Eq.(12) describes the dependence of the total number of states below some energy on the total number of particles in the box. But, as explained in section III, for an ideal FES gas, the Haldane exclusion property must hold locally in energy space, so the state counting result (12) is a necessary but not sufficient condition. The strategy to prove that the box Hamiltonian (11) indeed describes an ideal FES gas, is to first map the interacting system onto a non-interacting one in terms of renormalized quantum numbers (pseudo-energies) which display the exclusion statistics property. Then we can use standard counting arguments to arrive at the expression (5) for the entropy of an ideal FES gas.

\footnotetext{
${ }^{1}$ In going from (11) to (11) it is important to correctly incorporate the effect of Bose statistics [21. There is an extra factor of 2 in the interaction term in the Heisenberg equation for the quantum field operator as compared to the similar looking Gross-Pitaevskii equation for a classical Bose field. This also implies an extra factor of two in the mean field one-body Hamiltonian (11), and here we differ from the treatment in 13.
} 
The starting point is the total energy, $E_{\ell}$ which is not simply the sum of the one particle energies $\epsilon_{i}^{\ell}$ of (11), but given by

$$
E_{\ell}=\sum_{i}\left[\epsilon_{i}^{\ell}-\frac{\pi \hbar^{2}}{m} g n\left(\vec{r}_{\ell}\right)\right]
$$

where the last term compensates for the double counting of the interaction energy. Recalling from above that the number of states below a given energy is proportional to the corresponding kinetic energy, we can rewrite Eq.(14) as

$$
E_{\ell}=\sum_{i=1}^{N_{\ell}}\left(V\left(\vec{r}_{\ell}\right)+\frac{2 \pi \hbar^{2}}{m b^{2}}\left[k_{i}+\frac{g}{2} N_{\ell}\right]\right)
$$

where $k_{i}=d_{\ell, i}$ is the number of available single-particle states below the energy of particle $i$, and we choose to order the labelling of particles in a given microstate such that $0 \leq$ $k_{1} \leq k_{2} \cdots \leq k_{N_{\ell}}$. Although the energy levels (the integers $\left\{k_{i}\right\}$ ) are not equally spaced, we assume that the box is large enough for this effect to be negligible.

Next introduce the quantities $\tilde{k}_{i}$ by

$$
\tilde{k}_{i}=k_{i}+g \sum_{j} \theta\left(\tilde{k}_{j}-\tilde{k}_{i}\right)
$$

with $\theta(x)$ being the step function, and rewrite the energy as a sum of "pseudo-energies", $\tilde{\epsilon}_{i}^{\ell}$,

$$
\begin{aligned}
E_{\ell} & =\sum_{i=1}^{N_{\ell}} \tilde{\epsilon}_{i}^{\ell} \\
\tilde{\epsilon}_{i}^{\ell} & =V\left(r_{\ell}\right)+\frac{2 \pi \hbar^{2}}{m b^{2}} \tilde{k}_{i} .
\end{aligned}
$$

Note that, expressed in terms of these pseudo-energies, the system is formally noninteracting. The interaction term $\sim g N_{\ell}$ has been absorbed in the renormalized quantum numbers $\tilde{k}_{i}$. The exclusion properties of this system are now manifest since (16) implies that the pseudo-energies must satisfy,

$$
\tilde{\epsilon}_{i+1}^{\ell} \geq \tilde{\epsilon}_{i}^{\ell}+\frac{2 \pi}{m b^{2}} \hbar^{2} g
$$

As mentioned above, the relation (12) holds only because the kinetic energy and the number density scale as the same power of the cell size $b$. This is true for the present case of particles with quadratic dispersion in two dimensions, but also for particles in one dimension with linear dispersion, which is the case for anyons in the lowest Landau level, or equivalently, chiral particles on a circle with an $N^{2}$ type interaction [9]. In fact, the two models studied in this paper and in [9] can be exactly mapped onto each other by identifying the $\tilde{k}_{i}$ in (16) with the "pseudo-momenta" introduced in [9].

The proof that the exclusion property (18) corresponds to an ideal FES gas as defined in [15], amounts to showing that the (non-equilibrium) entropy of our system equals that of an ideal FES gas. This derivation is a straightforward modification of the one given in 22] 
for a multispecies system in the fermionic representation: Going to a continuum description with "momenta" $p_{i}$ and "pseudo-momenta" $\tilde{p}_{i}$ defined as

$$
p_{i}=\left(\frac{2 \pi \hbar}{b}\right)^{2} k_{i} ; \quad \tilde{p}_{i}=\left(\frac{2 \pi \hbar}{b}\right)^{2} \tilde{k}_{i}
$$

replacing the sums over $k$ and $\tilde{k}$ by integrals in the usual way and denoting the corresponding particle densities in momentum space by $\nu(p)$ and $\rho(\tilde{p})$, respectively, one finds the continuum version of Eq.(16),

$$
\tilde{p}=p+g \int d \tilde{p}^{\prime} \rho\left(\tilde{p}^{\prime}\right) \theta\left(\tilde{p}-\tilde{p}^{\prime}\right)
$$

Furthermore one has to demand conservation of the number of particles when changing variables from $p$ to $\tilde{p}$, i.e. $\nu(p) d p=\rho(\tilde{p}) d \tilde{p}$. Combining this with Eq.(20) gives

$$
\nu(p)=\frac{\rho(\tilde{p})}{1-g \rho(\tilde{p})} .
$$

Inserting this into the standard expression for the bosonic non-equilibrium entropy,

$$
S=-k\left(\frac{b}{2 \pi \hbar}\right)^{2} \int d p[\nu \ln \nu-(1+\nu) \ln (1+\nu)]
$$

exactly reproduces the continuum version of the FES entropy (5)

$$
S=-k\left(\frac{b}{2 \pi \hbar}\right)^{2} \int d \tilde{p}[\rho \ln \rho-(1-(g-1) \rho) \ln (1-(g-1) \rho)+(1-g \rho) \ln (1-g \rho)],
$$

from which all thermodynamics follows.

\section{BEYOND MEAN FIELD THEORY: QUANTUM MECHANICS}

So far our analysis was entirely in the context of mean field approximations. It is an interesting question whether the full quantum problem of a two dimensional gas with a delta function interaction also allows a description in terms of exclusion statistics in some range of temperatures. In this section we show that this is indeed the case, by computing the correction to the second virial coefficient due to the interaction, and showing that in a certain parameter regime, the result is consistent with Eq.(8). To this end, we first calculate the quasi-2D scattering solution with a delta function potential to find the s-wave scattering phase shift. The corresponding shift in the pressure of the gas can be found from the BethUhlenbeck formula 23] which relates the second virial coefficient to the scattering phase shifts. Although the interaction naively does not involve any dimensionful parameter, it is known that a pure delta function interaction gives rise to short distance singularities and requires a renormalization of the interaction strength which introduces a renormalization

scale and thus breaks scale invariance [24]. The two-dimensional s-wave scattering phase shift $\delta_{0}$ is given by 25 


$$
\cot \delta_{0}=-\frac{4}{\pi g}+\frac{2}{\pi} \ln \left(\frac{p l_{z}^{e f f}}{\hbar}\right)
$$

where $p$ is the relative momentum in the two body scattering process, and $g$ is related to the (three dimensional) scattering length by (2). Note that the phase shift does depend on the momentum $p$ via the renormalization scale, $l_{z}^{e f f}$ which up to a numerical factor equals the transverse extent $l_{z}$ of the quasi $2 \mathrm{~d}$ system. The Beth-Uhlenbeck formula then gives the following shift in the pressure due to interactions [26],

$$
\Delta P=-\left(n \lambda_{T}\right)^{2} \frac{2 k T}{\pi} \int_{0}^{\infty} d p e^{-\frac{p^{2}}{m k T}} \frac{d \delta_{0}}{d p} .
$$

In general, the integral in (25) is a function of $l_{z} / \lambda_{T}$, but in the relevant parameter range,

$$
a \ll l_{z} \ll \lambda_{T},
$$

it is approximately constant, as can be seen by the following argument: First partially integrate and note that the phase shift vanishes at $p=0$. For small $g$, i.e. $a \ll l_{z}$ we consider the $O(g)$ correction to the ideal Bose gas:

$$
\frac{d \Delta P}{d g}=-\frac{4}{m \pi}\left(n \lambda_{T}\right)^{2} \int_{0}^{\infty} d p p e^{-\frac{p^{2}}{m k T}} \frac{d \delta_{0}}{d g} .
$$

where

$$
\frac{d \delta_{0}}{d g}=-\frac{4}{\pi g^{2}}\left[1+\left(-\frac{4}{\pi g}+\frac{2}{\pi} \ln \left(p l_{z}^{e f f} / \hbar\right)\right)^{2}\right]^{-1}
$$

Next note that the integral has a high momentum cutoff at $p \sim \frac{\hbar}{\lambda_{T}}$ due to the exponential. This cutoff suppresses contributions from values of $p$ large enough for the logarithm to give a sizable correction, since we have to demand $l_{z} \ll \lambda_{T}$ in order for the system to be effectively two-dimensional. For small $p$, the contribution from the logarithm is suppressed for small enough $g$, i.e. by demanding $\exp (1 / g) \gg \lambda_{T} / l_{z}$. Neglecting the logarithmic dependence in the integral in (27), one finds $\frac{d \delta_{0}}{d g} \approx-\frac{\pi}{4}$. Thus, in the parameter range (26) there is no dependence on the scale $l_{z}$, and the Gaussian integral can be performed to get the following result for the leading order in $g$ correction to the pressure,

$$
\Delta P=\frac{1}{2} k T\left(n \lambda_{T}\right)^{2} g
$$

in perfect agreement with (10). We can thus conclude that for temperatures and couplings in the range (26) the scattering approach is consistent with the mean field approximation used in the previous section. It is an open question whether there are any corrections to higher virial coefficients.

\footnotetext{
${ }^{2}$ This range might be experimentally relevant in the near future [27].
} 


\section{DISCUSSION}

Since we have explicitly ignored both the possibility of a quantum condensate and of pairing fields other than the density, the results of this paper (and those of Ref. [13]) can not be used for temperatures below or in the vicinity of the Bose condensation transition $T_{c}$. This is true irrespective of whether this transition is of the Kosterlitz-Thouless type or not [25]. Rather our results should be relevant in a temperature regime where the exclusion statistics, due to the repulsive interaction, corresponds to a small correction to the ideal Bose gas. Moreover we have seen that one has to demand $a \ll l_{z} \ll \lambda_{T}$ in order for the scattering approach to be consistent with the mean field results. Since this sets a lower limit on the thermal wave length it also implies that the mean field approach can not be trusted for very rapidly changing potentials even at high temperatures.

It is an interesting open question whether the quasi particles above a two-dimensional Bose condensate also can be described using exclusion statistics. To answer this question one would analyze the corresponding statistical mechanics in a more sophisticated mean field approximation that includes effects of phase coherence and pairing mean fields [21].

Acknowledgement: S.V. thanks Prof. Christophe Salomon for a discussion on possible experiments. 


\section{REFERENCES}

[1] The Quantum Hall Effect, eds. R.E. Prange and S.M. Girvin, (Second edition, Springer Verlag, New York 1990).

[2] C. L. Kane, L. Balents and M. P. A. Fisher, Phys. Rev. Lett. 79, 5086 (1997); R. Egger and A. Gogolin, Phys. Rev. Lett. 79, 5082 (1997).

For a recent review, see R. Egger et al. in Interacting Electrons in Nanostructures, edited by R. Haug and H. Schoeller (Springer) (cond-mat/0008008).

[3] J.M. Leinaas and J. Myrheim, Nuovo Cimento 37 B, 1 (1977).

[4] F.D.M. Haldane, Phys. Rev. Lett. 67, 937 (1991).

[5] S. B. Isakov, Int. J. Mod. Phys. A 9, 2563 (1994).

[6] Z. N. C. Ha, Phys. Rev. Lett. 73, 1574 (1994);

Z. N. C. Ha, Nucl. Phys. B 435, 604 (1995);

M. V. N. Murthy and R. Shankar, Phys. Rev. Lett. 73, 3331 (1994).

[7] A. Dasnières de Veigy and S. Ouvry, Phys. Rev. Lett. 72, 600 (1994); D. Li and S. Ouvry, Nucl. Phys. B 430, 563 (1994).

[8] M. D. Johnson and G. S. Canright, Phys. Rev. B 49, 2947 (1994); S. He, X.-C. Xie, and F.-C. Zhang, Phys. Rev. Lett. 68, 3460 (1992).

[9] T. H. Hansson, J. M. Leinaas and S. Viefers, Nucl. Phys. B 470, 291 (1996).

[10] T. H. Hansson, J. M. Leinaas and S. Viefers, Phys. Rev. Lett. 86, 2930 (2001).

[11] T. Haugset and H. Haugerud, Phys. Rev. A 57, 3809 (1998).

[12] L. P. Pitaevskii and A. Rosch, Phys. Rev. A 55, R853 (1997); W. J. Mullin, J. Low Temp. Phys. 106, 615 (1997); ibid 110, 167 (1998).

[13] R. K. Bhaduri, S. M. Reimann, S. Viefers, A. Ghose Choudhury and M. K. Shrivastava, J. Phys. B 33, 3895 (2000).

[14] R. K. Bhaduri, M. V. N. Murthy, and M. K. Shrivastava, Phys. Rev. Lett. 76, 165 (1996).

[15] S. B. Isakov, Mod. Phys. Lett. B 8, 319 (1994).

[16] Y. S. Wu, Phys. Rev. Lett. 73, 922 (1994).

[17] S. B. Isakov, D. P. Arovas, J. Myrheim, and A. P. Polychronakos, Phys. Lett. A 212, 299 (1996).

[18] C. Nayak and F. Wilczek, Phys. Rev. Lett. 73, 2740 (1994).

[19] S.B. Isakov, S. Mashkevich and S. Ouvry, Nucl. Phys. B 448, 457 (1995).

[20] D. Sen, Nucl. Phys. B 360, 397 (1991); S. Viefers, F. Ravndal, and T. Haugset, Am. J. Phys. 63, 369 (1995).

[21] A. Griffin, Phys. Rev. B 53, 9341 (1996).

[22] S. B. Isakov and S. Viefers, Int. J. Mod. Phys. A 12, 1895 (1997).

[23] See e.g. L. D. Landau and E. M. Lifshitz, Statistical Physics (Pergamon Press, London,1954) p. 236 for 3D. The modification to $2 \mathrm{D}$ is straightforward.

[24] This is a well defined quantum problem, see e.g. R. Jackiw in M. A. B. Bég Memorial Volume (World Scientific, Singapore, 1991).

[25] D. S. Petrov, M. Holzmann, and G. V. Shlyapnikov, Phys. Rev. Lett. 84, 2551 (2000).

[26] P. Giacconi, F. Maltoni and R. Soldati, Phys. Rev. B 53, 10065 (1996).

[27] C. Salomon, private communication. 